\title{
Lentivirus-mediated knockdown of rhomboid domain containing 1 inhibits colorectal cancer cell growth
}

\author{
JUNYI HAN ${ }^{1,2^{*}}$, JUNCHAO BAI ${ }^{3 *}$, YAO YANG $^{2}$, HUA YIN $^{2}$, WEI GAO ${ }^{2}$, AIGUO LU $^{2}$, FEI LIU $^{1}$, \\ HAIYAN GE ${ }^{2}$, ZHONGMIN LIU ${ }^{4}$, JINYI WANG ${ }^{4}$ and LAN ZHONG ${ }^{1}$
}

\begin{abstract}
Departments of ${ }^{1}$ Gastroenterology and ${ }^{2}$ General Surgery, Shanghai East Hospital, Tongji University, Shanghai 200120;
${ }^{3}$ Department of General Surgery, People's Hospital of Dayao, Yunnan $675400 ;{ }^{4}$ Department of Thoracic Surgery, Shanghai East Hospital, Tongji University, Shanghai 200120, P.R. China
\end{abstract}

Received January 16, 2014; Accepted August 12, 2014

DOI: $10.3892 / \mathrm{mmr} .2015 .3365$

\begin{abstract}
Rhomboid domain containing 1 (RHBDD1), is a member of the rhomboid protease family, which has a pivotal role in the progression of numerous severe malignancies. However, its role in colorectal carcinoma (CRC) remains to be elucidated. In the present study, RHBDD1 was shown to be widely expressed in CRC cell lines. Lentivirus-mediated RNA interference was employed to knockdown RHBDD1 expression in RKO CRC cells. Functional analyses indicated that depletion of RHBDD1 expression resulted in significantly reduced $\mathrm{CRC}$ cell proliferation and colony formation, and induced a G0/G1 phase cell cycle arrest. The findings of the present study suggest that RHBDD1 may contribute to CRC tumorigenesis and serve as a potential therapeutic target in human CRC.
\end{abstract}

\section{Introduction}

Malignant colorectal cancer or carcinoma (CRC) originates from the epithelial cells of the colon or rectum. CRC is associated with a high risk of cancer morbidity and mortality and a previous genomic analysis demonstrated that colorectal and rectal cancers have considerably similar patterns (1). CRC is responsible for $\sim 500,000$ deaths annually (2), and in developed countries, one out of three cases of CRC are fatal (3). Genetic studies have resolved the regulation of cellular metabolism, proliferation, differentiation and survival in CRC (4). However, further research is required to fully understand the molecular changes associated with the pathophysiology of CRC. The effector molecules and signal transduction

Correspondence to: Professor Lan Zhong or Professor Junyi Han, Department of Gastroenterology, Shanghai East Hospital, Tongji University, 150 Jimo Road, Shanghai 200120, P.R. China

E-mail: lanzhongdr@163.com

E-mail: junyidr@163.com

${ }^{*}$ Contributed equally

Key words: RHBDD1, colorectal carcinoma, RNAi, proliferation pathways responsible for the development of CRC remain to be elucidated.

Previous studies have indicated that conserved intramembrane proteolytic mechanisms are associated with the regulation of cellular processes, including transcriptional control, growth factor secretion and apoptosis $(5,6)$. It has been well demonstrated that intramembrane proteases are involved in critical cellular processes, including apoptosis and signaling transduction (6-10). The rhomboid serine proteases are expressed in most species, and have been observed in both bacteria and humans (11), they are considered to be one of the most-widely conserved membrane proteases (12). The first rhomboid serine protease was initially identified in a Drosophila genetics study, where it was shown to activate the upstream epidermal growth factor receptor (EGFR) signaling pathway (13). Previous research has also demonstrated that numerous yeast rhomboid proteases have a role in mitochondrial membrane remodeling (14).

Rhomboid domain containing 1 (RHBDD1), a mammalian rhomboid protease highly expressed in testis, has previously been identified as a pro-apoptotic member of the B-cell lymphoma 2 family (15). RHBDD1 has been shown to be highly expressed in chronic myeloid leukemia patients, as compared with healthy controls (16). RHBDD1 exhibited a proteolytic activity in the tumor suppressor activated pathway-6, in both HCT116 and RKO colon cancer cells (15). Downregulation of RHBDD1 also demonstrated the ability to suppress proliferation and colony formation capability of HepG2 hepatocellular carcinoma cells (17). However, the precise function of RHBDD1 in CRC progression remains unclear. In the present study, RHBDD1 was confirmed to be highly expressed in numerous CRC cell lines. To determine the role of RHBDD1 in human CRC, a lentivirus-mediated short hairpin RNA (shRNA) was used to knockdown RHBDD1 expression in RKO CRC cells. The effects of RHBDD1 expression knockdown on CRC cell growth were then investigated.

\section{Materials and methods}

Cell Culture. SW480, SW620, RKO, DLD-1, HCT116 and HT-29 human CRC cell lines and HEK293T human embryonic kidney cells were obtained from the Cell Bank of the Chinese 
Academy of Sciences (Shanghai, China). SW480, SW620, RKO and DLD-1 cells were cultured in RPMI-1640, supplemented with $10 \%$ fetal bovine serum (FBS; HyClone Laboratories Inc., Logan, UT, USA). HCT116 and HT-29 cells were cultured in McCoy's 5A media, supplemented with 10\% FBS (Sigma-Aldrich, St. Louis, MO, USA). HEK293T cells were cultured in Dulbecco's modified Eagle's medium, supplemented with 10\% FBS (HyClone Laboratories Inc.).

Construction of recombinant lentivirus. The small interfering RNA (siRNA) sequence for RHBDD1 (NM_032276) (5'-GCTGGGATTCTTGTTGGACTA-3') was screened and validated, to confirm its use as the candidate siRNA. A non-silencing siRNA sequence (5'-CCAAGGAAGTGCAA TTGCATA-3') was used as a control. shRNAs corresponding to both the RHBDD1 and control siRNA sequences, were synthesized as 21-nt inverse repeats, separated by a 9-nt loop for each sequence and inserted downstream of the U6 promoter in the pFH-L lentiviral vector (Shanghai Hollybio Co., Ltd., Shanghai, China). The lentiviruses were generated by triple transfection of $80 \%$ confluent HEK293T cells, with modified pFH-L plasmid, and helper vectors pVSVG-I and pCMV $\Delta$ R8.92 (Shanghai Hollybio Co., Ltd.), using Lipofectamine ${ }^{\circledR} 2000$ (Invitrogen Life Technologies, Carlsbad, CA, USA). The lentiviruses were harvested in serum-free medium after three days, filtered and concentrated using primed Centricon ${ }^{\circledR}$ Plus-20 filter devices (Millipore, Billerica, MA, USA).

RNA extraction and quantitative polymerase chain reaction $(q P C R)$. RKO cells were pre-cultured and infected with the recombinant lentivirus for five days. Total RNA was extracted using TRIzol ${ }^{\circledR}$ reagent (Gibco Life Technologies, Carlsbad, CA, USA), according to the manufacturer's instructions. Total RNA $(5 \mathrm{mg})$ was reverse transcribed to produce the first strand of cDNA, using $200 \mathrm{U} / \mathrm{ml}$ SuperScript II RT (Invitrogen, Carlsbad, CA, USA). RHBDD1 mRNA expression levels were evaluated by qPCR using a Bio-Rad Connect Real-Time PCR platform (Bio-Rad Laboratories, Hercules, CA, USA) with SYBR Green PCR core reagents (Bio-Rad Laboraties); $\beta$-actin was used as an internal reference. The following primers were used: RHBDD1 forward, 5'-GCAGGACTGAGTGAAGAAGAAC-3', and reverse, 5'-GTGAGAGATGAAACCCGTAGG-3'; and $\beta$-actin forward, 5'-GTGGACATCCGCAAAGAC-3' and reverse, 5'-AAAGGGTGTAACGCAACTA-3'. The RT-qPCR analysis was performed with the following amplification steps: initial denaturation at $95^{\circ} \mathrm{C}$ for $1 \mathrm{~min}$, followed by 40 cycles of denaturation at $95^{\circ} \mathrm{C}$ for $5 \mathrm{sec}$ and annealing extension at $60^{\circ} \mathrm{C}$ for $20 \mathrm{sec}$. The results are presented as cycle threshold $(\mathrm{Ct})$ values, the threshold PCR cycle number at which the amplified product is first detected. The average $\mathrm{Ct}$ was calculated for both RHBDD1 and $\beta$-actin, and the $\triangle \mathrm{CT}$ was determined as the mean of the triplicate $\mathrm{Ct}$ values for RHBDD1, minus the mean of the triplicate $\mathrm{Ct}$ values for $\beta$-actin.

Western blot analysis. RKO cells, five days after lentiviral infection, were lysed in 2X SDS sample buffer [100 mM Tris-Hcl (pH 6.8), 10 mM EDTA, 4\% SDS, 10\% glycine]. The proteins $(3 \mu \mathrm{g})$ were loaded onto a polyacrylamide gel and were separated by SDS-PAGE, followed by transfer to polyvinylidene difluoride membranes (Millipore). The blots were blocked with TBST containing 5\% non-fat dry milk at room temperature for $1 \mathrm{~h}$ and then incubated with primary antibodies: rabbit anti-RHBDD1 (1:500dilution; Sigma-Aldrich) and mouse anti-GAPDH(1:3,000 dilution; Santa Cruz Biotechnology, Inc., Dallas, TX, USA) at $4^{\circ} \mathrm{C}$ overnight. The blots were then probed with antibodies against RHBDD1 (1:500 dilution; Sigma-Aldrich) and mouse anti-GAPDH, (1:3,000 dilution; Santa Cruz Biotechnology, Inc.). Following washing three times (5 min each) with TBST, the blots were incubated with the corresponding horseradish peroxidase-conjugated secondary antibodies: goat anti-rabbit IgG (1:5,000 dilution; Santa Cruz Biotechnology, Inc.) and goat anti-mouse IgG (1:5,000 dilution; Santa Cruz Biotechnology, Inc.) at room temperature for $2 \mathrm{~h}$. The blots were then visualized using a Super Enhanced Chemiluminescence Detection Reagent (Applygen Technologies Inc., Beijing, China).

Colony formation assay. RKO cells were cultured in 24-well plates and treated with the recombinant lentiviruses at a multiplicity of infection (MOI) of 20. Following a $72 \mathrm{~h}$ incubation, the cells were washed, re-cultured in the prepared 6-well plates (400 cells/well) and allowed to form natural colonies. Eight days later the cells in both groups were subjected to Giemsa staining. Briefly, the cells were washed and fixed using paraformaldehyde, the fixed cells were then washed twice with phosphate-buffered saline (PBS), treated with Giemsa (Sigma-Aldrich) for $10 \mathrm{~min}$, washed three times with double-distilled $\mathrm{H}_{2} \mathrm{O}$, and then photographed using a digital camera. The number of colonies $(>50$ cells/colony) were counted.

MTT Viability Assay. RKO cells were cultured in 24-well plates and inoculated with either RHBDD1-shRNA or control-shRNA lentiviruses, at a MOI of 20 . Following a $72 \mathrm{~h}$ incubation, the cells were washed, re-cultured in the prepared 96-well plates (2,000 cells/well) and the cell viability was analyzed using MTT reagent (Sigma-Aldrich) and acidic isopropanol (10\% SDS, $5 \%$ isopropanol and $0.01 \mathrm{~mol} / \mathrm{l} \mathrm{HCl}$ ).

Flow cytometric analysis. RKO cells were harvested by centrifugation at $404 \mathrm{xg}$ for $5 \mathrm{~min}, 72 \mathrm{~h}$ following infection. The pellets were washed twice with cold PBS, fixed with cold $70 \%$ ethanol, centrifuged and resuspended with PBS. The pellets were washed twice with cold PBS, fixed with cold $70 \%$ ethanol at $4^{\circ} \mathrm{C}$ overnight and then resuspended in propidium iodide/RNase/PBS for incubation in the dark (room temperature for $30 \mathrm{~min}$ ). The suspensions were filtered through a 400-mesh membrane and subjected to cell cycle analysis using a flow cytometer (BD Biosciences, San Jose, CA, USA). (BD Biosciences, San Jose, CA, USA).

Statistical analysis. All statistical analyses were performed using SPSS version 13.0 (SPSS, Inc., Chicago, IL, USA)software. The differences between the groups were compared using a Student's t-test, and data were expressed as the means \pm standard deviation of triplicate experiments. A $\mathrm{P}<0.05$ was considered to indicate a statistically significant difference.

\section{Results}

Knockdown of RHBDDl expression by siRNA. Preliminary studies were performed to determine the prime candidate 
A
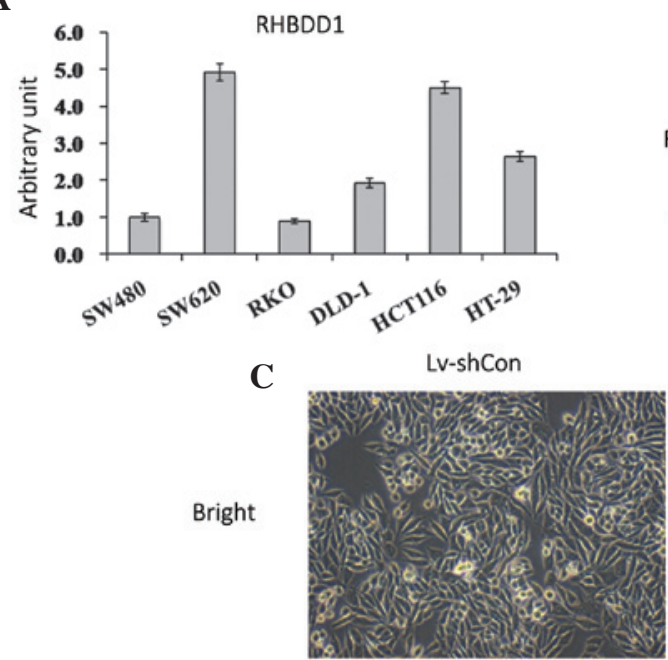

GFP

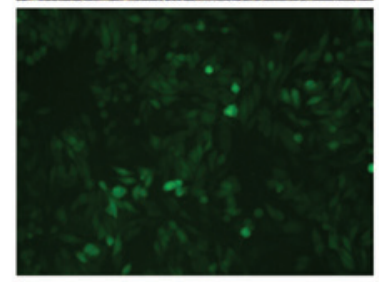

D

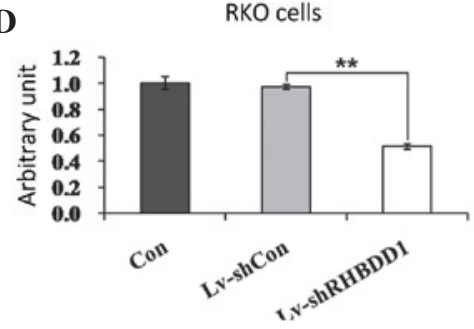

B

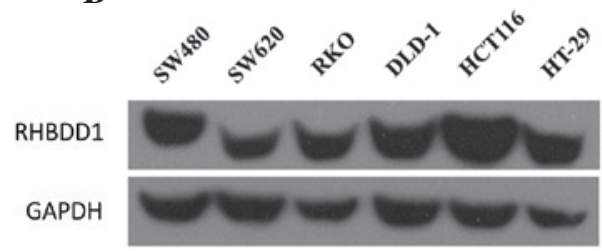

Lv-shRHBDD1
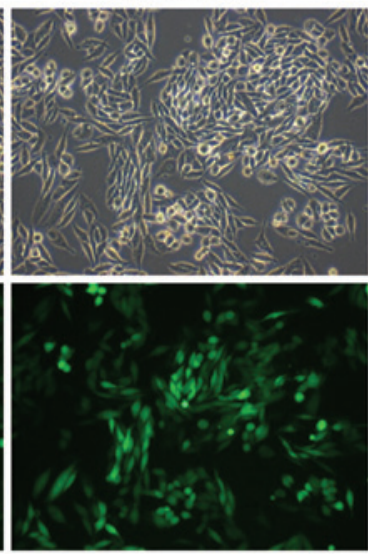

Figure 1. Downregulation of rhomboid domain containing 1 (RHBDD1) by lentivirus-mediated small hairpin (sh)RNA. (A) Quantitative polymerase chain reaction of RHBDD1 mRNA in six colon cancer cell lines. (B) Western blot analysis of RHBDD1 protein in six colon cancer cell lines. (C) Representative graphs of RKO cells infected with lentivirus at multiplicity of infection of 20 (magnification, x400). (D Expression analysis of RHBDD1 mRNA in RKO colorectal cancer cells with three treatments (control, Lv-shCon, Lv-shRHBDD1) by qPCR. (E) Expression analysis of RHBDD1 protein in RKO cells with three treatments (control, Lv-shCon, Lv-shRHBDD1) by western blotting. Con, uninfected; Lv-shCon, control lentivirus with non-silencing; Lv-shRHBDD1, RHBDD1-silencing lentivirus; GFP, green fluorescent protein. ${ }^{* *} \mathrm{P}<0.01$, as compared to treatment with Lv-shCon.

CRC cell line for further in vitro studies. The mRNA and protein expression levels of RHBDD1 were analyzed in six CRC cell lines: SW480, SW620, RKO, DLD-1, HCT116 and HT-29. As shown in Fig. 1A and B, RHBDD1 was widely expressed in all of the cell lines tested. The RKO cell line, which had moderate RHBDD1 expression levels, is commonly used in colon cancer studies due to its high proliferation rate and low coefficient of MOI. Therefore, the following in-depth in vitro investigations were conducted using $\mathrm{RKO}$ cells. To investigate the role of RHBDD1 in human CRC, control (Lv-shCon) and RHBDD1-shRNA (Lv-shRHBDD1), lentiviruses were constructed. RKO cells were cultured and infected with either the Lv-shCon or Lv-shRHBDD1 lentivirus. The images were photographed following a $72 \mathrm{~h}$ incubation. An embedded green fluorescent protein-tag was used to visualize the transfection efficiency of the lentiviruses. As shown in Fig. 1C, $>80 \%$ of the cells were successfully infected with the recombinant lentiviruses. The specificity and efficiency of the RHBDD1 RNA interference (RNAi) treatment was further
RKO cells

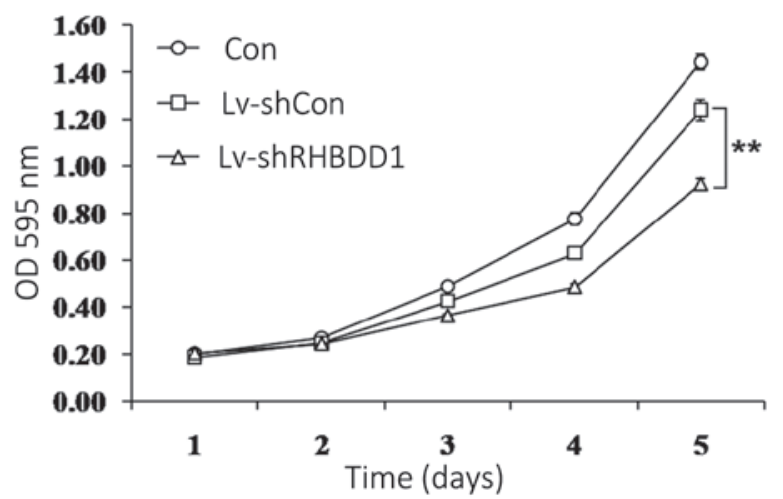

Figure 2. Knockdown of rhomboid domain containing 1 (RHBDD1) suppresses RKO colorectal cancer cell proliferation. The growth curve of RKO cells with three treatments was determined by MTT assay. OD, optical density; Con, uninfected; Lv-shCon, control lentivirus with non-silencing; Lv-shRHBDD1, RHBDD1-silencing lentivirus. ${ }^{* *} \mathrm{P}<0.01$, as compared to treatment with $\mathrm{Lv}$-shCon. 

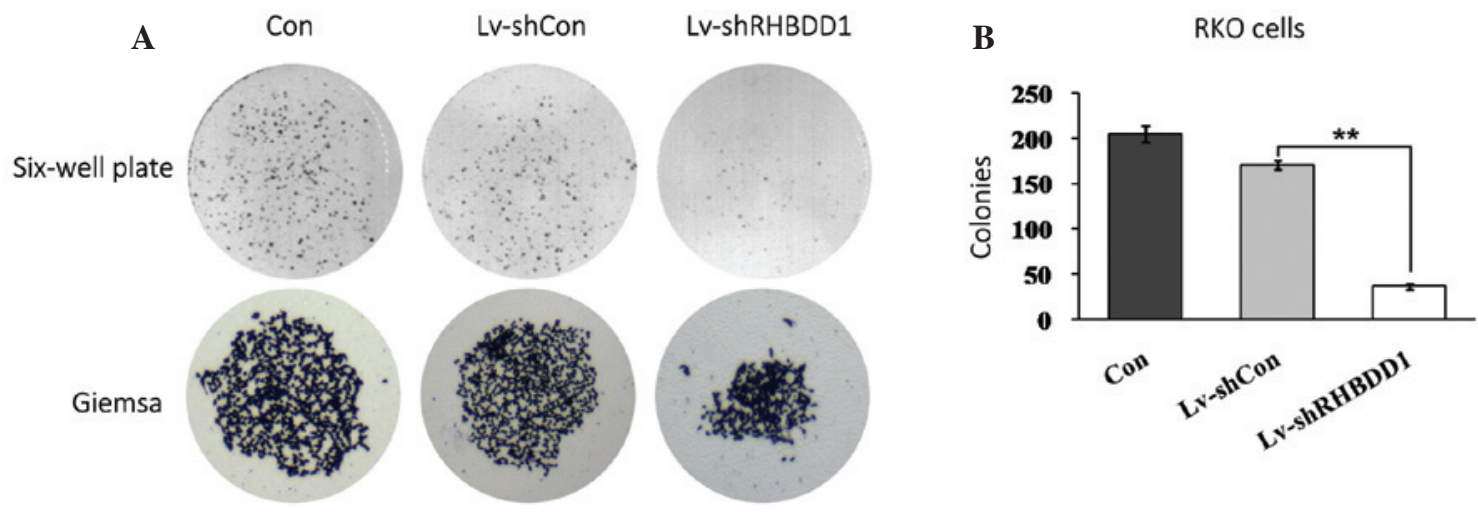

Figure 3. Rhomboid domain containing 1 (RHBDD1) knockdown reduces colony formation capacity of RKO colorectal cancer cells. (A) Representative images of colonies captured by a light microscopy. (B) Statistical analysis of the number of colonies with Gimesa staining in RKO cells with three treatments. Con, uninfected; Lv-shCon, control lentivirus with non-silencing; Lv-shRHBDD1, RHBDD1-silencing lentivirus ${ }^{* * *} \mathrm{P}<0.01$, as compared to treatment with Lv-shCon.

A

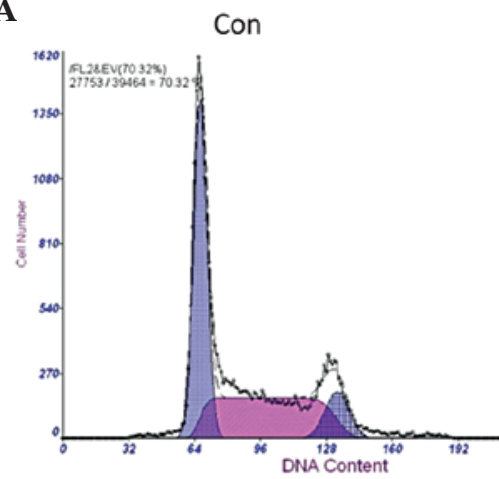

Lv-shCon

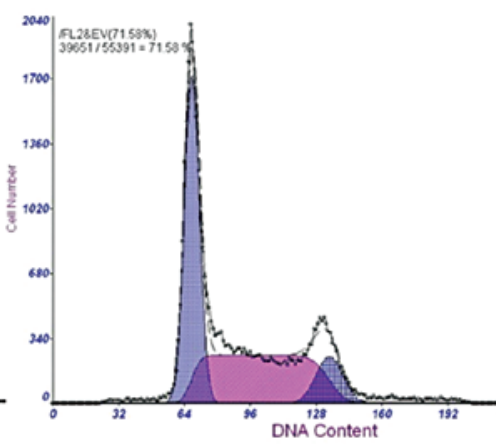

Lv-ShRBDD1
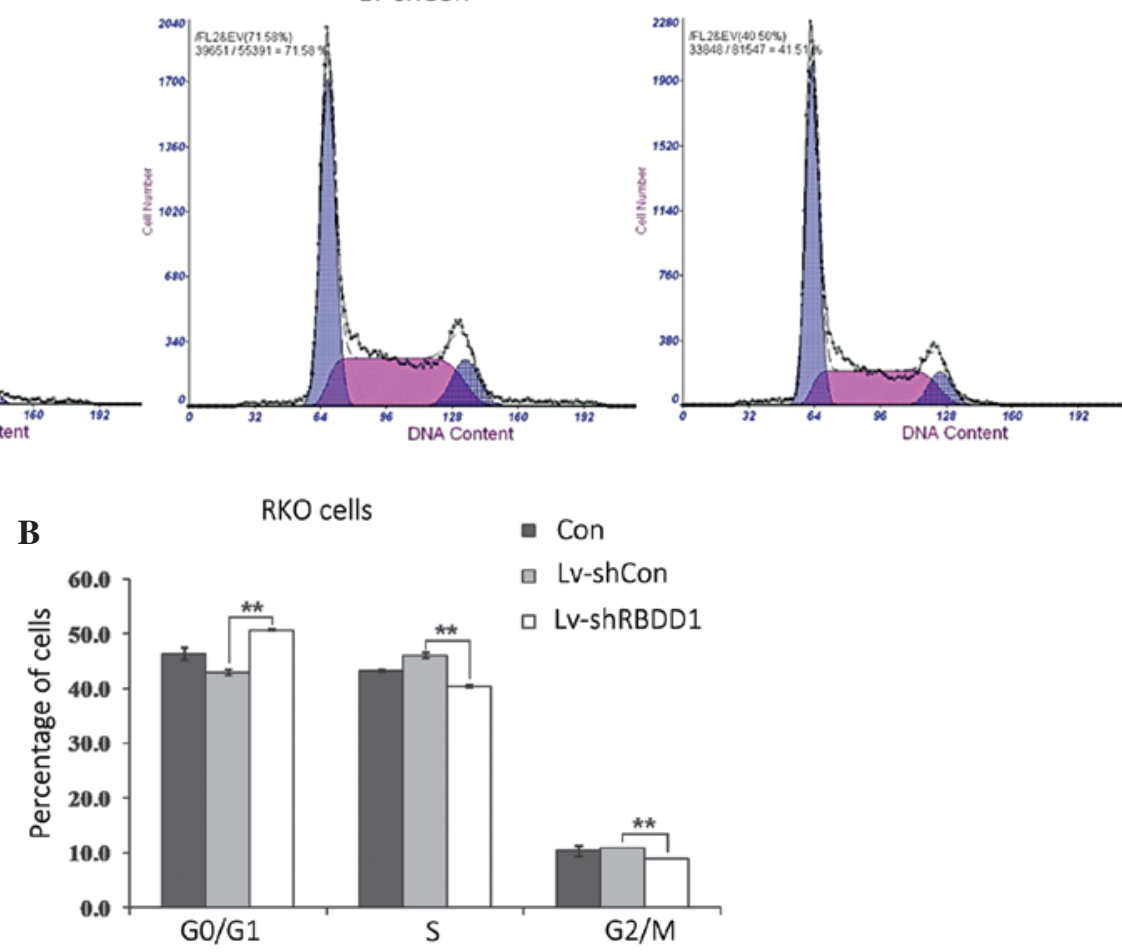

Figure 4. Knockdown of rhomboid domain containing 1 (RHBDD1) blocks cell cycle progression. (A) Representative images of cell cycle distribution by flow cytometric analysis. (B) Knockdown of RHBDD1 in RKO ovarian cancer cells induced cell cycle arrest in G0/G1 phase at $72 \mathrm{~h}$ after lentivirus infection. Con, uninfected; Lv-shCon, control lentivirus with non-silencing; Lv-shRHBDD1, RHBDD1-silencing lentivirus. * $\mathrm{P}<0.01$, as compared to treatment with Lv-shCon.

verified as the control lentivirus, inserted with one irrelevant sequence, had no impact on RHBDD1 translation (Fig. 1D). The target lentivirus Lv-shRHBDD1 markedly downregulated the endogenous RHBDD1 mRNA expression levels, as compared with the control lentivirus $(\mathrm{P}<0.01)$. Knockdown efficiency was further confirmed using western blot analysis, there was little RHBDD1 protein expression detected following RHBDD1 specific knockdown by RNAi (Fig. 1E).

Knockdown of RHBDDI inhibits RKO cell proliferation. To explore the role of RHBDD1 in CRC tumorigenesis, the proliferation of RKO cells following RHBDD1 expression knockdown, was analyzed using an MTT assay. As shown in
Fig. 2, the proliferation rate of Lv-shRHBDD1 infected cells was markedly lower, as compared with the Lv-shCon infected and uninfected cells $(\mathrm{P}<0.01)$. These results indicate that knockdown of RHBDD1 expression may inhibit RKO cell proliferation.

Knockdown of RHBDDI suppresses colony formation of RKO cells. The colony forming capacity of RKO cells was determined using a monolayer cell culture. As shown in Fig .3A, knockdown of RHBDD1 expression markedly suppressed colony formation in RKO cells, as determined by a colony formation assay. The number of colonies formed in RKO cells infected with Lv-shRHBDD1, was significantly decreased, as compared with the Lv-shCon infected and uninfected cells (Fig. 3B, p<0.01). 
These results indicate that RHBDD1 may have an important role in the cell growth and tumorigenesis of CRC.

Knockdown of RHBDD1 induces G0/G1 phase arrest in RKO cells. To elucidate whether RHBDD1 knockdown induced cell growth inhibition by affecting the progression of the cell cycle, flow cytometric analysis was performed in RKO cells. As shown in Figure 4, following infection with Lv-shRHBDD1, an increased number of cells accumulated at the G0/G1 phase and the percentage of cells in the $\mathrm{S}$ and $\mathrm{G} 2 / \mathrm{M}$ phases were reduced $(\mathrm{P}<0.01)$. These results indicate that $\mathrm{RHBDD} 1$ knockdown may inhibit the growth of RKO cells via cell cycle arrest.

\section{Discussion}

Since the initial identification of rhomboid proteases (18), there have been indications that they may have a fundamental function within numerous cell types. However little research has been performed to determine their role in CRC. Vertebrate rhomboid genes are grouped into three classes and HBDD1 is classed as an active cellular rhomboid. Previous research has demonstrated that in Drosophila that rhomboid proteases regulate EGFR signaling pathways (19,20), and that rhomboid families may have pivotal roles in the modulation of EGFR transactivation (21). The monoclonal antibodies Cetuximab and Panitumumab, which target EGFR, have been effective against CRC, in clinical practice (22). However, it remains unclear as to whether the rhomboid protease family is involved in CRC progression.

A previous study in hepatoma cells suggested that RHBDD1 may be a positive regulator for HCC cell growth and apoptosis using recombinant lentivirus-mediated silencing of RHBDD1 in HepG2 cells (17). It is therefore conceivable that RHBDD1 has a similar, essential role in CRC tumorigenesis. In the present study, we noted that RHBDD1 was widely expressed in numerous human CRC cell lines. To illuminate the functional role of RHBDD1 in the CRC cells, the present study used lentivirus-mediated siRNA to silence the expression of RHBDD1 in the RKO colon cancer cells. Knock down of RHBDD1 expression was found to markedly suppress cell proliferation and colony formation in the RKO cells. Furthermore, flow cytometric analysis revealed that depletion of RHBDD1 in the RKO cells led to cell cycle arrest in the G0/G1 phase.

A previous study from a murine model indicated that RHBDD1 has anti-apoptotic potential and that spermatogonia GC-1 cells, a mouse derived spermatogonia line, were more sensitive to apoptotic stimuli following knock down of RHBDD1 expression (23). Further investigation is required to elucidate the modulation of RHBDD1 on CRC cell apoptosis and its regulatory mechanism in CRC development and progression.

In conclusion, the findings of the present study demonstrated that RHBDD1 could promote CRC cell growth via cell cycle control. RHBDD1 may serve as a potential therapeutic target in human CRC.

\section{Acknowledgements}

The authors of the present study are grateful for the financial support received from the Academic Leaders Training Program of Pudong Health Bureau of Shanghai (no. PEWd2010-05).

\section{References}

1. Cancer Genome Atlas Network: Comprehensive molecular characterization of human colon and rectal cancer. Nature 487: 330-337, 2012.

2. Merika E, Saif MW, Katz A, Syrigos K and Morse M: Review. Colon cancer vaccines: an update. In Vivo 24: 607-628, 2010.

3. Cunningham D, Atkin W, Lenz HJ, et al: Colorectal cancer. Lancet 375: 1030-1047, 2010.

4. Fearon ER: Molecular genetics of colorectal cancer. Annu Rev Pathol 6: 479-507, 2011.

5. Lemberg MK: Intramembrane proteolysis in regulated protein trafficking. Traffic 12: 1109-1118, 2011.

6. Brown MS, Ye J, Rawson RB and Goldstein JL: Regulated intramembrane proteolysis: a control mechanism conserved from bacteria to humans. Cell 100: 391-398, 2000.

7. Kroos L and Yu YT: Regulation of sigma factor activity during Bacillus subtilis development. Curr Opin Microbiol 3: 553-560, 2000.

8. Weihofen A and Martoglio B: Intramembrane-cleaving proteases: controlled liberation of proteins and bioactive peptides. Trends Cell Biol 13: 71-78, 2003.

9. Freeman M: Rhomboid proteases and their biological functions. Annu Rev Genet 42: 191-210, 2008.

10. Wolfe MS and Kopan R: Intramembrane proteolysis: theme and variations. Science 305: 1119-1123, 2004.

11. Urban S: Rhomboid proteins: conserved membrane proteases with divergent biological functions. Genes Dev 20: 3054-3068, 2006.

12. Koonin EV, Makarova KS, Rogozin IB, Davidovic L, Letellier MC and Pellegrini L: The rhomboids: a nearly ubiquitous family of intramembrane serine proteases that probably evolved by multiple ancient horizontal gene transfers. Genome Biol 4: R19, 2003.

13. Wasserman JD,Urban S and Freeman M: A family of rhomboid-like genes: Drosophila rhomboid-1 and roughoid/rhomboid-3 cooperate to activate EGF receptor signaling. Genes Dev 14: 1651-1663, 2000.

14. McQuibban GA, Saurya S and Freeman M: Mitochondrial membrane remodelling regulated by a conserved rhomboid protease. Nature 423: 537-541, 2003.

15. Wang Y, Guan X, Fok KL, et al: A novel member of the Rhomboid family, RHBDD1, regulates BIK-mediated apoptosis. Cell Mol Life Sci 65: 3822-3829, 2008.

16. Lin YN, Gui FM, Shen H, et al: Expression of RHBDD1 gene in patients with chronic myeloid leukemia and its clinical significance. Zhongguo Shi Yan Xue Ye Xue Za Zhi 21: 12-15, 2013 (Chinese).

17. Liu XN, Tang ZH, Zhang Y, et al: Lentivirus-mediated silencing of rhomboid domain containing 1 suppresses tumor growth and induces apoptosis in hepatoma HepG2 cells. Asian Pac J Cancer Prev 14: 5-9, 2013.

18. Urban S and Dickey SW: The rhomboid protease family: a decade of progress on function and mechanism. Genome Biol 12: 231, 2011.

19. Lee JR, Urban S, Garvey CF and Freeman M: Regulated intracellular ligand transport and proteolysis control EGF signal activation in Drosophila. Cell 107: 161-171, 2001.

20. Urban S, Lee JR and Freeman M: Drosophila rhomboid-1 defines a family of putative intramembrane serine proteases. Cell 107: 173-182, 2001.

21. Zou H, Thomas SM, Yan ZW, Grandis JR, Vogt A and Li LY: Human rhomboid family-1 gene RHBDF1 participates in GPCR-mediated transactivation of EGFR growth signals in head and neck squamous cancer cells. FASEB J 23: 425-432, 2009.

22. Normanno N, Tejpar S, Morgillo F, De Luca A, Van Cutsem E and Ciardiello F: Implications for KRAS status and EGFR-targeted therapies in metastatic CRC. Nat Rev Clin Oncol 6: 519-527, 2009.

23. Wang Y, Song W, Li S, et al: GC-1 mRHBDD1 knockdown spermatogonia cells lose their spermatogenic capacity in mouse seminiferous tubules. BMC Cell Biol 10: 25, 2009. 